Ann. Génét. Sél. anim., 1978, 10 (2), 233-250

\title{
On the heredity of water intake and feed efficiency in the Fowl
}

\author{
A. Bordas, A. Obeidah*, P. MǴ́RAT \\ Laboratoire de Génétique Factorielle \\ I.N.R.A. - C.N.R.Z. \\ 78 Jouy-en-Josas
}

\section{Summary}

This study was carried out on two populations, "M99" and „Jouy ", during five consecutive years, mainly to determine the genetic variation of water intake and water/feed ratio and the effect of water/feed ratio on feed intake. The main results can be summarized as follows:

I. - A recessive major gene in the two strains seems responsible for "polydipsia" in some individuals. There is a large mean difference for water intake between the two distinguished genotypes, didi for excessive water intake and the normal genotypes designated by (Di). birds.

The mean value for water/feed ratio for didi hens proved to be about double that for (Di)

2. - The variability in water/feed ratio is essentially due to water intake.

3. - "Polydipsic " birds have a significantly higher " residual" feed consumption (after taking account of body weight and egg production) than " normal" ones.

4. - There is no significant difference between the two genotypes for egg number and egg weight.

5. - Some phenotypic correlations may be modified by the presence of the didi genotype.

\section{Introduction}

Several workers have, recently, studied the phenomenon of excessive water intake by hens housed in cages during the productive period (see review by LEESON et al., I976). SUNDE (cf BRETH, I964) showed the importance of water as a limiting factor of other physiological functions. Besides, the convenience of selecting poultry populations with a scarce water content in their excrements is considered necessary for good management. It would be interesting to demonstrate the

${ }^{*}$ ) permanent address : Deptof Animal Breeding, Cairo Univer sity, CAIRo, EGYPT. 
existence of correlated factors for selection on this character which is uneasy to measure directly on large numbers.

As in many studies of genetic factors, an adequate measurement of the trait requires considerable attention. Water intake is closely correlated with food intake and factors affecting food intake indirectly influence water consumption (ZEIGLER et al., I97I; BIERER et al., I966). Accordingly to assess any influence increased water intake may have on feed intake, water/feed ratio was considered as a determining criterion in two populations studied during the last five years.

The objective of this work is to point out the existence in our populations of two genotypes namely (di) for excessive water intake and (Di) for normal water intake. This may be worthy for indirect selection, and to shorten the period required to perform the phenotypic classification of the flock.

\section{Material and methods}

\section{1. - Experimental populations and traits measured}

Two populations have been studied: an experimental flock segregating for several marker genes ("Jouy " strain, years I970, I97I, I975) and a laying-type Rhode-Island red strain originating from the "Station Experimentale d'aviculture du Magneraud ", of which a subline was pedigree bred at Jouy-en-Josas from I970 on (" M99" Strain, years r97I to I975).

TABLE I

Symbol and description of the characters used

Symboles et description des caractères

\footnotetext{
WI Water intake (g./day, average on I4 days)

p. roo $\frac{W I}{B W}$ p. Ioo $\frac{\text { Water intake }}{\text { Body weight }}$

W Average body weight during control of feed (g.)

$\Delta \mathrm{W}$ Variation in body weight $/ 28$ days (g.) average of 3 periods

E Total egg mass $/ 28$ days (g.), average of 3 periods

F Feed intake $/ 28$ days (g.), average of 3 periods

$\mathrm{R}$ Deviation of observed feed intake from the regression on body weight, egg mass and variation of body weight $/ 28$ days (see BORDAS and MÉRAT, I974)

$R^{\prime} \quad$ Deviation from the regression on body weight and egg mass $/ 28$ days

$\frac{W I}{\mathrm{~F}}$ Water/feed ratio (daily mean values)

SM Sexual maturity (days)

EN Egg number (sexual maturity to ro mths age)

EW Average egg weight (g) at Io months age on 4 successive eggs

$\mathrm{HU}$ Haugh Units at ro months age on 4 successive eggs

STH Shell thickness (o.oI $\mathrm{mm}$ ) at ten months age on 4 successive eggs

WL Wattle length $(\mathrm{cm})$ at ten months age

SL Shank length $(\mathrm{cm})$ at ten months age
} 
The hatching period (two hatches two weeks apart) was in spring for the “ M99 " strain and autumn for " Jouy" strain.

In each year chicks were raised on floor till the age $\mathrm{x} 6$ weeks. Then a sample of females (59 to 94 according to year) obtained from several sire and dam families were set in individual cages. Several laying - and egg traits were recorded on this sample. Moreover, from about 8 months age, feed intake of each bird (with a $16 \mathrm{p}$. Ioo protein and $2520 \mathrm{kcal} / \mathrm{Kg} \mathrm{ME}$ ration (or I0.55 $\mathrm{MJ} / \mathrm{kg}$ ), the formula being the same for all years) was controlled during 3 successive 28 day periods. During I 4 consecutive days in the last period, individual water consumption was also measured (including possible waste of water).

For each individual, only its average value is considered for the traits of which the measurement is repeated, and only birds having all measurements recorded are considered.

Table I summarizes the definition of the observed traits and abbreviated symbols designating them in the following tables.

\section{2. - Test of major gene hypothesis for water intake: definition of genotypes and tests}

In each examined year for the two populations, some individuals with abnormally high water consumption appeared. From preliminary analysis the water/ feed ratio (on the basis of daily means for each bird) was considered the most discriminating between individuals and is used here.

Each year the distribution of the sample for this ratio was obviously asymmetric. About ro to $I_{5}$ p. Ioo of the individuals had values exceeding (quite appreciably for most of them) the mean of the whole sample augmented by twice its standard deviation. The average value of these " abnormal " birds was about double that of the other (" normal ") birds, of which the distribution seemed approximately normal.

This suggested the presence of two distinct populations for the trait studied, possibly corresponding to different genotypes at a " major" locus.

To check further this possibility, in each year from each strain the mean value $\bar{x}$ and standard deviation $s$ of the whole sample for water/feed ratio were estimated. Individuals with a value exceeding $\bar{x}+2 s$ were considered “ abnormals " and excluded from the distribution. After this exclusion, a new mean $\bar{x}_{1}$ and standard deviation $s_{1}$ were computed. If in the corresponding sample there remained a few individuals with value appreciably superior to $\bar{x}_{1}+2 s_{1}$, these individuals were also considered " abnormals " and excluded from the distribution of " normal " birds of which the new mean $\bar{x}_{2}$ and the new standard deviation $s_{2}$ were calculated. It was never necessary to go further, as $\bar{x}_{2}$ and $s_{2}$ were always close respectively to $\bar{x}_{1}$ and $s_{1}$.

The value $\bar{x}_{2}+2 s_{2}$ (or $x_{1}+2 s_{1}$ ) finally separated two groups of individuals, those with a lower value (" normals ") and those with a higher value (" abnormals "). The normality of the distribution of the two groups separated in this way was tested. Then the genetic hypothesis of a major gene acting on the trait studied was checked by comparing, within full-sib families containing at least one " abnormal " hen, the observed proportion of " abnormals " to that expected, (chi-square test) taking account of the frequency of the postulated genotypes and of the limited family size. 


\section{3. - Statistical analysis on quantitative traits}

Pairs of fu1l sisters were formed, one " normal ", the other " abnormal " (or " polydipsic") according to the criterion described above.

From all pairs formed in each population, the mean value of " normals " and " polydipsics" is compared for each trait by a t-test (pair method). On the other hand, an analysis of variance is drawn from the same data with " genotype " (normal vs polydipsic) and year as sources of variation.

The same data allow, finally, estimating the phenotypic correlation coefficients between all variables within each genotype after testing homogeneity of the correlations between populations.

Anticipating on the following, as " normal " birds may be either homozygote for the supposed " major " gene (DiDi) or heterozygote (Didi) they will be designated in abbreviated way as (Di), and accordingly (di) for polydipsic. The term "genotype" will be used for each of these two groups.

\section{Results}

\section{1. - Presence and identification of a major gene for " polydipsia"}

Table 2 gives the numbers and average values for the water/feed ratio of " normals" and " abnormals" (polydipsics) by year and strain.

Figure I shows, on pooled years and strains, the distribution for the same trait expressed in deviations from means by strain and year, for " normals " and " abnormals", considering only families containing both types of individuals (part overlapping of the two distributions comes from the pooling of several years; according to the procedure used for definition of the two groups these is no overlapping within years). Within these families the total numbers of " nor.mal" and " abnormal " birds are respectively 72 and 37 in the "Jouy" strain, 85 and 43 for the "M99" and 157 and 80 for both together.

\section{2. - Mean perfarmance of genotypes and correlations within genotype}

Tabłes 3,4 and 5 contain the mean values, $t$ tests and analyses of variances for each population and " genotype " for water intake, feed intake, water/feed ratio, egg mass, gain in body weight, $R$ and $R^{\prime}$.

Tables $6,7,8$ point out the mean values, $t$ tests and analyses of variance by year and " genotype " for each population for sexual maturity, egg number, egg weight, Haugh units, shell thickness, wattle and shank length.

Table 9 shows the pooled estimates, on a within-strain and year basis, of the correlations between the above mentioned characters within each genotype.

The significances of the differences between the correlation values within genotypes are presented in table Io, with the overall estimates of correlations for the two "genotypes". 
TABLE 2

Numbers of " normal " and " abnormal" hens and mean values for water /feed ratio Nombre de poules "normales " et " anormales" et valeurs moyennes pour le rapport eau /aliment

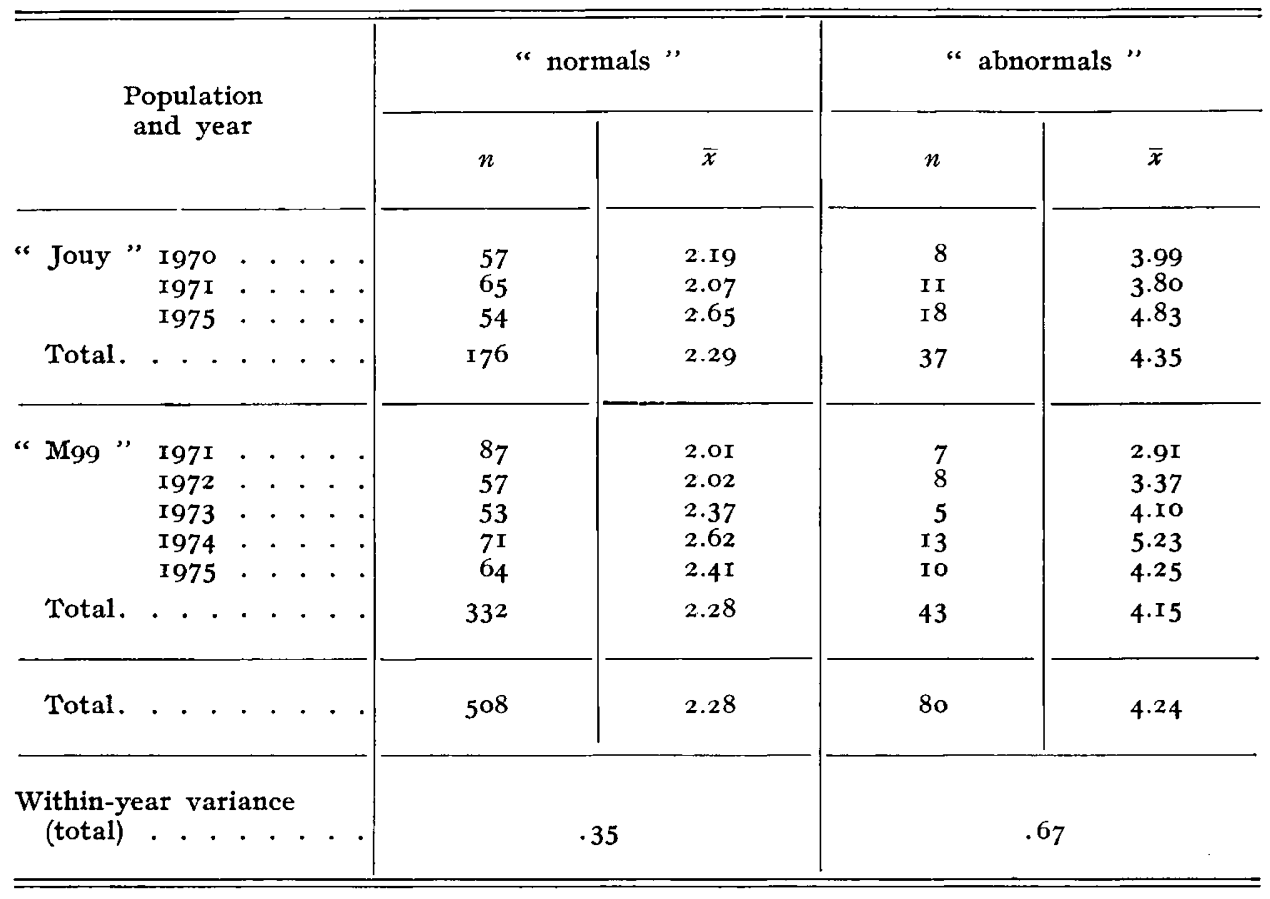

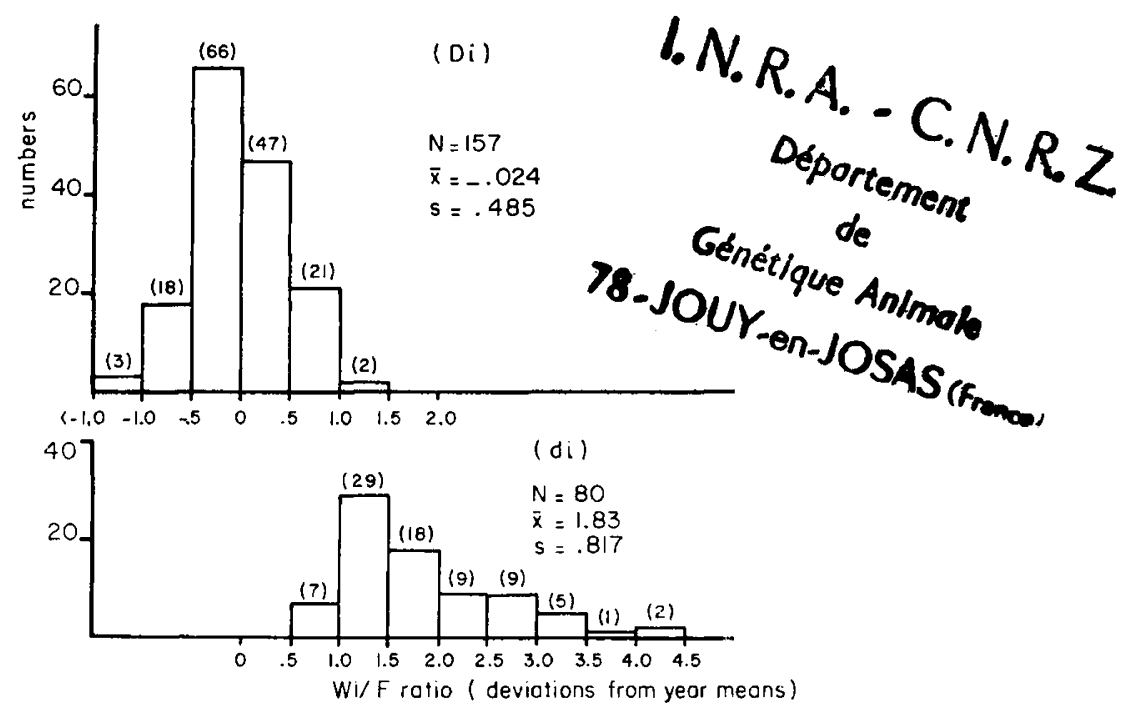

FIG. I. - Distribution of water/feed ratio for $(D i)$ and (di) birds (years pooled). Distribution du rapport eau/aliment pour les poules (Di) et (di) (années groupées) 


\section{TABLE 3}

Means for water and feed consumption and associated traits with respect to the two groups ("genotypes") (di) and (Di)

Moyennes de la consommation d'eau et d'aliment et des caractères associés pour les deux génotypes (di) et $(D i)$

a) Jouy Population

\begin{tabular}{|c|c|c|c|c|c|c|c|c|c|c|}
\hline Year & Gén. & WI & $\% \frac{\mathrm{WI}}{\mathrm{B}} \frac{\mathrm{W}}{\mathrm{B}}$ & $\vec{W}$ & $\Delta W$ & $\mathrm{E}$ & $\mathbf{F}$ & $\mathrm{R}$ & $\mathbf{R}^{\prime}$ & $\frac{W I}{F}$ \\
\hline \multirow{2}{*}{ I970. . } & (di) & $534 \cdot I$ & 33.67 & 2104.2 & I 6.7 & $9^{8} 5 \cdot 3$ & 3682.1 & I 89.7 & 240.6 & 4.05 \\
\hline & (Di) & 299.6 & 21.06 & 2164.2 & 40.0 & 975.8 & 3590.8 & $-6 . I$ & 115.6 & 2.35 \\
\hline \multirow{2}{*}{ I97I. $\cdot$} & (di) & 542.9 & 23.44 & 2260.2 & 83.6 & $95^{2.3}$ & 3987.9 & I 89.9 & 366.3 & 3.87 \\
\hline & (Di) & 298.5 & 12.32 & 2288.4 & II3.6 & $\longdiv { 1 0 5 0 . 6 }$ & 3895.6 & $-8 \mathrm{I} \cdot 5$ & 158.0 & 2.14 \\
\hline \multirow[b]{2}{*}{$1975 \cdot}$. & (di) & 635.9 & 28.60 & 2254.3 & 43.2 & I 025.2 & 3777.0 & -10.0 & $87 \cdot 3$ & 4.75 \\
\hline & (Di) & 323.0 & 14.96 & 2 I 86.5 & 36.8 & $\overline{1021.7}$ & 3716.2 & -8.4 & 78.2 & 2.43 \\
\hline \multirow{2}{*}{$\begin{array}{l}\text { overall }\left(^{*}\right) \text {. } \\
\text { means } . .\end{array}$} & (di) & 582.0 & 28.29 & 2218.6 & 48.9 & 992.9 & 3817.7 & I 02.0 & 210.9 & $4 \cdot 3 I$ \\
\hline & (Di) & 309.7 & I 5.70 & 2212.1 & 6I.I & rorg.r & 3739.7 & -30.2 & I 11.9 & 2.32 \\
\hline
\end{tabular}

$\left({ }^{*}\right) \mathbf{N}=36$ pairs.

b) M99 population

\begin{tabular}{|c|c|c|c|c|c|c|c|c|c|c|}
\hline Year & Gen. & WI & $\% \frac{\mathrm{WI}}{\mathrm{BW}}$ & $\overline{\mathrm{W}}$ & $\Delta \mathrm{W}$ & $\mathrm{E}$ & $\mathrm{F}$ & $\mathbf{R}$ & $\mathrm{R}^{\prime}$ & $\frac{\mathrm{WI}}{\mathrm{F}}$ \\
\hline \multirow{2}{*}{$197 \mathrm{I}$} & (di) & $345 \cdot 5$ & I7.9I & I 948.3 & 34.9 & I 073.8 & 3566.0 & 94.9 & 207.9 & 2.73 \\
\hline & (Di) & 184.5 & 10.77 & I 717.9 & -6.0 & 878.1 & 2914.0 & $7 \times .9$ & $-9 \mathrm{r} .2$ & I. 79 \\
\hline \multirow{2}{*}{1972} & (di) & 449.6 & 23.28 & I 947.2 & 48.0 & 964.2 & 3373.2 & 54.2 & 221.8 & 3.73 \\
\hline & (Di) & 204.0 & $9 \cdot 7^{2}$ & $2 \mathrm{I} 48.4$ & $4^{22.0}$ & $74^{2.4}$ & 3176.8 & -28.4 & II 8.0 & 1.82 \\
\hline \multirow{2}{*}{1973} & (di) & 5 or.6 & 24.76 & 2039.0 & 6.2 & I 093.6 & 3592.0 & $\mathrm{r} 69.2$ & I 77.2 & $3.9 \mathrm{I}$ \\
\hline & (Di) & $227 . \mathrm{I}$ & 10.96 & $2 I 15.0$ & 86.2 & 920.2 & 3376.0 & $-5 \mathrm{I} \cdot 5$ & 59,0 & I. 88 \\
\hline \multirow{2}{*}{1974.} & (di) & 477.0 & 23.93 & 2059.3 & -40.0 & 756.6 & 2594.0 & -47.9 & $-\mathrm{I} 64 . \mathrm{I}$ & 5.73 \\
\hline & (Di) & 228.9 & 10.89 & 2080.7 & 40.0 & $\longdiv { 1 0 4 7 . 4 }$ & 3094.0 & -113.7 & 2.5 & 2.05 \\
\hline \multirow{2}{*}{ I975 } & (di) & 423.9 & 21.39 & I 975.0 & 94.6 & 644.2 & 2894.0 & 68.6 & 296.5 & 4.24 \\
\hline & (Di) & 225.3 & I I.I 6 & 2008.0 & 76.8 & $651 \cdot 3$ & 2993.0 & — 2.4 & I83.6 & 2.I 6 \\
\hline \multirow{2}{*}{$\begin{array}{l}\text { overall }\left({ }^{*}\right) \\
\text { means } .\end{array}$} & (di) & $429 . I$ & $2 \mathrm{r} .75$ & I 990.2 & 33.0 & $887 . x$ & $3 \mathrm{I} 75.2$ & 46.4 & I 52.8 & 4.03 \\
\hline & (Di) & 212.7 & 10.77 & I 983.4 & 44.5 & 840.3 & $3074 \cdot I$ & -53.3 & 49.5 & 1.95 \\
\hline
\end{tabular}

$(*) \mathrm{N}=35$ pairs. 


\section{TABLE 4}

The differences and " $\mathrm{t}$ " tests between the two genotypes fo water and feed consumption and associated traits

Différences entre les deux génotypes et tests $t$ pour la consommation d'aliment et d'eau et les caractères associés

a) Jouy population

\begin{tabular}{|c|c|c|c|c|c|c|c|c|c|c|}
\hline Year & $\bar{d} /(t t »$ & WI & $\% \frac{\mathrm{WI}}{\mathrm{BW}}$ & $\bar{W}$ & $\Delta \mathrm{W}$ & $\mathrm{E}$ & $\mathbf{F}$ & $\mathbf{R}$ & $\mathbf{R}^{\prime}$ & $\frac{\mathrm{WI}}{\mathrm{F}}$ \\
\hline \multirow[t]{2}{*}{ r970.. } & $\bar{d}$ & $234 \cdot 5^{I}$ & $\mathrm{I} 2.6 \mathrm{I}$ & -60.00 & -23.32 & 9.56 & $9 \mathrm{r} \cdot 33$ & I95.78 & 125.0 & I. 70 \\
\hline & $t$ & $6 . \mathrm{II}$ & $4 \cdot 75$ & 0.56 & 0.13 & 0.I3 & 0.72 & 2.20 & I.33 & $6.0 I$ \\
\hline \multirow{2}{*}{ I97I . . } & $\bar{d}$ & 244.44 & II.I 2 & -28.18 & $-29.9 \mathrm{I}$ & $-98.3^{6}$ & 92.27 & 271.36 & 208.37 & I. 73 \\
\hline & $t$ & 6.49 & 6.60 & 0.32 & 0.99 & I.04 & $0.5^{8}$ & 3.55 & I. 98 & $5 \cdot 73$ \\
\hline \multirow{2}{*}{ I975 . . } & $\bar{d}$ & $3 \times 2.88$ & I3.64 & 67.75 & $6.3^{8}$ & 3.50 & $60.8 \mathrm{I}$ & $-\mathrm{r} .59$ & 9.17 & 2.32 \\
\hline & $t$ & 14.19 & IO.II & $0.8 \mathrm{I}$ & 0.43 & 0.07 & $0.5^{6}$ & 0.03 & 0.13 & IO.I 4 \\
\hline \multirow{2}{*}{ overall $\left({ }^{*}\right)$} & $\vec{d}$ & 272.37 & 12.60 & $6.5^{\circ}$ & -I2.19 & $-26 . \mathrm{II}$ & 78.06 & $\mathrm{I} 32 . \mathrm{I} 6$ & 98.96 & I.99 \\
\hline & $t$ & $\begin{array}{r}14.80 \\
* * *\end{array}$ & $\begin{array}{r}\mathrm{T} 2.49 \\
* * *\end{array}$ & 0.12 & $\mathrm{I}, \mathrm{OI}$ & 0.64 & I.06 & $\begin{array}{r}2.85 \\
* *\end{array}$ & I.9I & $\begin{array}{r}\text { I2.53 } \\
* * *\end{array}$ \\
\hline
\end{tabular}

(*) 36 pairs.

b) M99 population

\begin{tabular}{|c|c|c|c|c|c|c|c|c|c|c|}
\hline Year & $\bar{d} / \ll t »$ & WI & $\% \frac{\mathrm{WI}}{\mathrm{BW}}$ & $\vec{W}$ & $\Delta W$ & $\mathrm{E}$ & $\mathbf{F}$ & $\mathrm{R}$. & $\mathrm{R}^{\prime}$ & $\frac{W I}{F}$ \\
\hline \multirow[t]{2}{*}{ I97I } & $\bar{d}$ & I60.97 & 7.14 & 230.44 & 40.89 & I95.67 & 651.89 & I 66.78 & $299.1 \mathrm{I}$ & 0.95 \\
\hline & $t$ & 22.47 & 10.66 & 4.13 & $\mathrm{x} .7 \mathrm{I}$ & 2.70 & $4.8 \mathrm{I}$ & 2.08 & $2.5 \mathrm{I}$ & 10.89 \\
\hline \multirow{2}{*}{ I972. . } & $\bar{d}$ & 245.60 & 13.56 & -20.12 & 6.00 & 221.80 & I96.40 & 82.60 & I03.80 & $\mathbf{I} .9 \mathbf{r}$ \\
\hline & $t$ & 4.78 & $5 \cdot 5^{2}$ & I.I6 & 0.25 & I.I2 & I.OI & $0.8 \mathrm{I}$ & 0.62 & $4 \cdot 94$ \\
\hline \multirow{2}{*}{ I973 . . } & $\bar{d}$ & $274 \cdot 36$ & I 3.80 & -76.00 & -80.00 & $\mathrm{I} 73.4^{\circ}$ & 216.00 & 220.72 & I 18.16 & 2.03 \\
\hline & $t$ & 3.64 & 3.69 & $0.7 \mathrm{I}$ & I.73 & $0.8 \mathrm{o}$ & 0.77 & $\mathrm{I} .7^{\circ}$ & 0.83 & 3.80 \\
\hline \multirow{2}{*}{ I974 . } & $\bar{d}$ & 248.10 & 13.04 & $-2 \mathrm{I} .43$ & -80.00 & -290.86 & -5.00 & 65.80 & $-\mathrm{x} 66.63$ & 3.68 \\
\hline & $t$ & 9. II & $9 \cdot 55$ & 0.24 & I. $5^{8}$ & I. 07 & I.I9 & 0.85 & 1.25 & $4 \cdot 7 \mathrm{I}$ \\
\hline \multirow{2}{*}{ x975.. } & $\bar{d}$ & I98.64 & 10.23 & $-33 \cdot 33$ & $\mathrm{I} 7.7^{8}$ & $7.1 \mathrm{r}$ & -98.89 & 70.99 & I 2.92 & 2.08 \\
\hline & $t$ & 14.68 & $13 \cdot 32$ & 0.39 & 0.55 & 0.03 & 0.36 & $0.7 \mathrm{I}$ & 0.87 & 5.92 \\
\hline \multirow{2}{*}{$\begin{array}{l}\text { overall } \\
\text { data }(*) . .\end{array}$} & $\bar{d}$ & $216.4^{\circ}$ & I0.98 & 6.80 & - I I.49 & 46.83 & IOI.II & I 7.63 & I03.3I & 2.08 \\
\hline & $t$ & $\begin{array}{r}\text { I } 4.29 \\
* * *\end{array}$ & $\begin{array}{r}\text { I } 3.45 \\
* * *\end{array}$ & 0.14 & 0.65 & 0.50 & 0.74 & $\begin{array}{r}2.80 \\
* *\end{array}$ & I.63 & $\begin{array}{l}8.36 \\
* * *\end{array}$ \\
\hline
\end{tabular}

(*) 35 pairs. totals within populations) 
A. BORDAS, A. OBEIDAH, P. MÉRAT

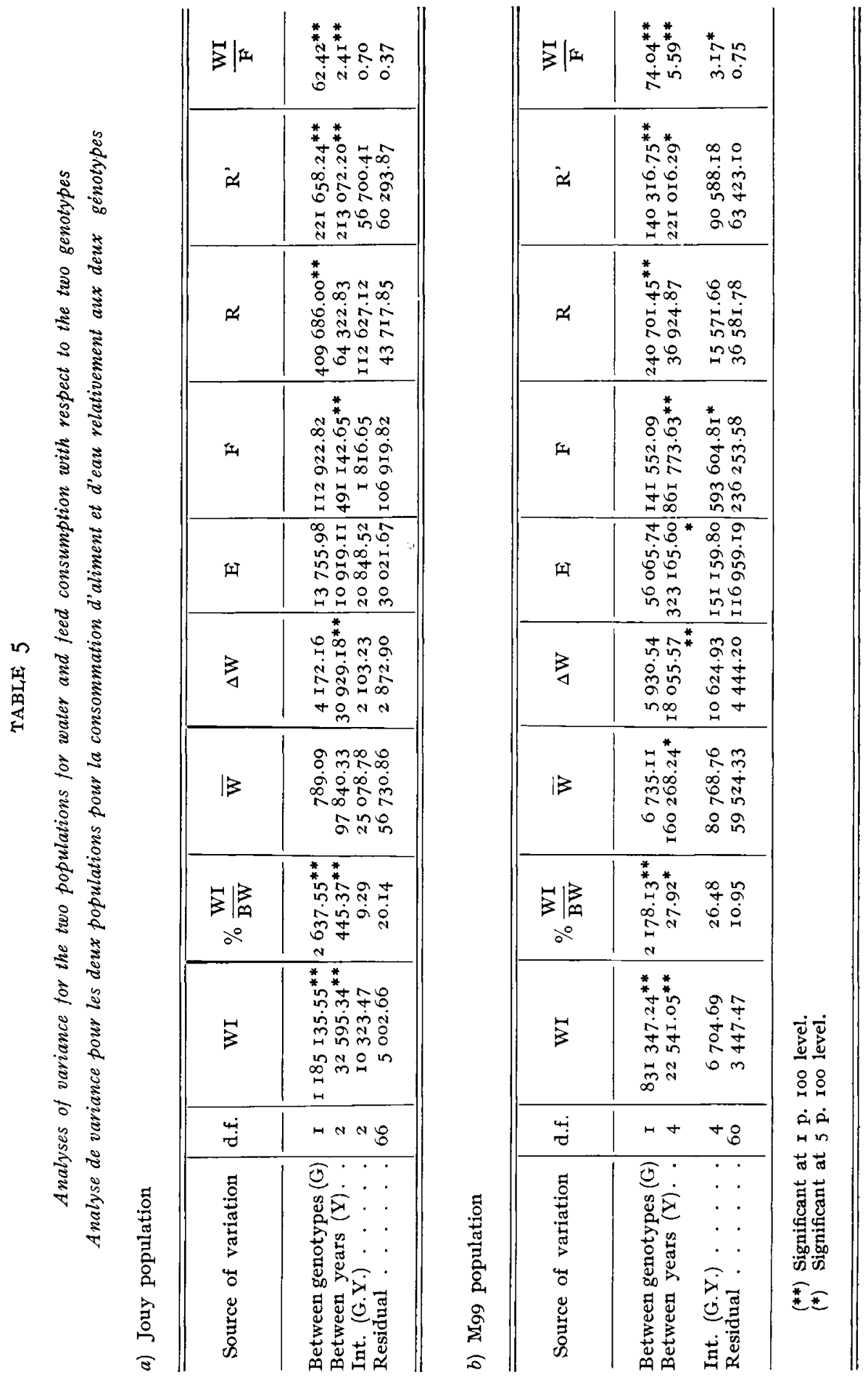


TABLE 6

Mean values for productive traits, egg traits and body measurements. with respects to the two genotypes in the two populations

Valeurs moyennes des caractères de production pour les deux génotypes dans les deux populations

a) Jouy population

\begin{tabular}{|c|c|c|c|c|c|c|c|c|c|c|c|c|}
\hline & Years & & & & Gen. & S.M. & E.N. & E.W. & H.U. & S.Th. & W.I. & S.L. \\
\hline \multirow[t]{2}{*}{ I970. } & \multirow{2}{*}{$\cdot \cdot \cdot$} & . & \multirow{2}{*}{\multicolumn{2}{|c|}{..}} & (di) & I 64.78 & 58.I & $5^{0.63}$ & 68.7 & 39.56 & 31.00 & I 06.44 \\
\hline & & & & & (Di) & I 60.78 & $58 \cdot 3$ & 49.99 & 71.0 & $3^{8.67}$ & 30.78 & 104.89 \\
\hline \multirow{2}{*}{ I97 I . } & \multirow{2}{*}{\multicolumn{4}{|c|}{$\cdot \cdot \cdot \cdot \cdot \cdot \cdot$}} & (di) & I 56.27 & 59.9 & 47.23 & 60.6 & $37 \cdot 18$ & 30.45 & $107 \cdot 36$ \\
\hline & & & & & (Di) & I 50.18 & 63.6 & 49.05 & 63.8 & 36.64 & 32.09 & I04.73 \\
\hline \multirow{2}{*}{ r975. } & \multirow[b]{2}{*}{ - } & \multirow{2}{*}{\multicolumn{2}{|c|}{$\cdot \cdot \cdot$}} & \multirow{2}{*}{ - $\cdot$} & (di) & I $67.5^{\circ}$ & 54.2 & 55.68 & 64.6 & 40.25 & 22.19 & II0.3I \\
\hline & & & & & (Di) & I 68.06 & 56.1 & 54.47 & $67 . \mathrm{I}$ & 40.31 & 29.44 & 109.63 \\
\hline \multirow{2}{*}{\multicolumn{2}{|c|}{ overall years }} & & & & (di) & 163.39 & 57.0 & 5 I. 83 & $64 \cdot 5$ & 39.14 & 27.14 & I 08.44 \\
\hline & & - & - & • & (Di) & I $60.7^{8}$ & 59.0 & 5 ז.69 & $67 . I$ & $3^{8.72}$ & $3^{0.58}$ & 106.94 \\
\hline
\end{tabular}

b) M99 population

\begin{tabular}{|c|c|c|c|c|c|c|c|c|}
\hline Years & Gen. & S.M. $\quad(*)$ & E.N. & E.W. & H.U. & S.Th. & W.L. & S.L. \\
\hline \multirow[t]{2}{*}{ I97I. } & (di) & $\mathbf{x} 68.00$ & 57.2 & 55.82 & 82.4 & 36.78 & 30.00 & I06.86 \\
\hline & (Di) & $183.5^{6}$ & 48.0 & $54.4^{6}$ & 90.2 & 34.67 & 26.78 & 104.II \\
\hline \multirow{2}{*}{ I972. } & (di) & & 51.2 & $56.5^{\circ}$ & 69.4 & 36.20 & 30.00 & 108.00 \\
\hline & (Di) & & $3^{8.2}$ & 58.96 & 82.6 & 38.00 & 28.20 & 109.80 \\
\hline \multirow{2}{*}{ I973. } & (di) & 149.60 & 97.0 & 54.00 & 69.4 & 36.40 & 31.20 & II $0.4^{\circ}$ \\
\hline & (Di) & 161.00 & 76.0 & 54.00 & 79.2 & 33.80 & 28.20 & 108.60 \\
\hline \multirow{2}{*}{ I 974 . } & (di) & I 73.86 & $57 . \mathrm{I}$ & 54.44 & 80.3 & 34.86 & 31.14 & I 12.30 \\
\hline & (Di) & $x 71.57$ & 80.4 & 54.96 & 76.9 & 34.57 & 28.00 & 106.30 \\
\hline \multirow{2}{*}{ I975. } & (di) & $\mathrm{r} 85.78$ & 37.9 & 58.09 & 77.0 & 37.00 & 26.89 & Iro.89 \\
\hline & (Di) & 185.78 & 41.0 & $57 . \mathrm{II}$ & 76.4 & 37.22 & 26.89 & I08.89 \\
\hline \multirow[b]{2}{*}{ overall years $(*)$} & (di) & I71.63 & 57.1 & 56.07 & 76.9 & 36.31 & 29.60 & $109.7^{\circ}$ \\
\hline & (Di) & I77.67 & $55 \cdot 3$ & 56.33 & $8 \mathrm{I} \cdot 3$ & 35.65 & $27 \cdot 50$ & 107.30 \\
\hline
\end{tabular}

(*) Four years only for sexual maturity. 


\section{TABLEAU 7}

$\mathrm{t}$ test between the two genotypes for production traits test $\mathbf{t}$ entre les deux génotypes pour les caractères de production

a) Jouy population

\begin{tabular}{|c|c|c|c|c|c|c|c|c|c|c|c|}
\hline & Years & & & $\bar{d} / \| t »$ & S.M. & E.N. & E.W. & H.U. & S.Th. & W.I. & S.L. \\
\hline \multirow{2}{*}{1970} & \multirow{2}{*}{\multicolumn{3}{|c|}{$\cdot \cdot \cdot \cdot \cdot \cdot \cdot \cdot \cdot$}} & $\bar{d}$ & 4.00 & -0.22 & 0.64 & -0.23 & 0.89 & 0.22 & I. 56 \\
\hline & & & & $t$ & 1.52 & 0.05 & 0.71 & 0.58 & 0.62 & 0.08 & 0.87 \\
\hline \multirow{2}{*}{ I97I . } & \multirow{2}{*}{\multicolumn{3}{|c|}{. . . . . . }} & $\bar{d}$ & 6.09 & -3.73 & 一 1.83 & -0.32 & 0.55 & $-\mathrm{I} .64$ & 2.64 \\
\hline & & & & $t$ & 0.94 & 0.89 & $0.7^{8}$ & I.I2 & 0.53 & I.I 2 & I.4I \\
\hline \multirow{2}{*}{ I975. } & \multirow{2}{*}{\multicolumn{3}{|c|}{$\cdot \cdot \cdot \cdot \cdot \cdot \cdot \cdot$}} & $\bar{d}$ & -0.56 & -I.94 & $\mathrm{r} .2 \mathrm{I}$ & -2.50 & -0.06 & -6.75 & 0.42 \\
\hline & & & & $t$ & 0.10 & 0.72 & I. I4 & 0.84 & 0.06 & 2.19 & 0.42 \\
\hline \multirow{2}{*}{\multicolumn{2}{|c|}{ overall years }} & \multirow{2}{*}{\multicolumn{2}{|c|}{$\cdot \cdot \cdot \cdot$}} & $\bar{d}$ & $2.6 \mathrm{I}$ & -2.10 & 0.14 & -0.26 & 0.42 & $-3 \cdot 44$ & $\mathrm{I} .5^{\circ}$ \\
\hline & & & & $t$ & 0.82 & I.04 & o.I 6 & $\mathrm{I} .42$ & 0.62 & $2.08^{*}$ & $x .5^{\circ}$ \\
\hline
\end{tabular}

b) M99 population

\begin{tabular}{|c|c|c|c|c|c|c|c|c|c|c|c|c|}
\hline & Year & & & & $\bar{d} / t$ & S.M. (I) & E.N. & E.W. & H.U. & S.Th. & W.L. & S.L. \\
\hline \multirow{2}{*}{ I97I. } & \multirow[b]{2}{*}{$\cdot$} & \multirow{2}{*}{\multicolumn{3}{|c|}{. . . . }} & $\ddot{d}$ & - I 5.56 & 9.22 & I. 37 & -0.78 & $2 . \mathrm{II}$ & 3.22 & 2.78 \\
\hline & & & & & $t$ & 2.66 & 2.87 & 0.87 & $2 . \mathrm{II}$ & $\mathrm{I.2 \textrm {I }}$ & I. 86 & 2.09 \\
\hline \multirow{2}{*}{ I972. } & \multirow{2}{*}{\multicolumn{2}{|c|}{$\cdot \cdot$}} & & \multirow{2}{*}{$\cdot$} & $\bar{d}$ & & 13,00 & -2.46 & -1.32 & $-\mathrm{I} .80$ & I. 80 & $-\mathbf{r} .80$ \\
\hline & & & $\cdot$ & & $t$ & & I. 45 & 0.92 & 2.59 & I.05 & I. 86 & $0.6 \mathrm{I}$ \\
\hline \multirow[b]{2}{*}{ I 973.} & \multirow[b]{2}{*}{$\cdot \cdot \cdot$} & \multirow{2}{*}{\multicolumn{3}{|c|}{$\cdot \cdot \cdot \cdot$}} & $\bar{d}$ & $-\mathrm{II} .4^{\circ}$ & 21.00 & 0.00 & -0.98 & 2.60 & 3.00 & I. 80 \\
\hline & & & & & $t$ & $\mathrm{I} .43$ & I. 47 & 0.00 & I. 29 & $\overline{\mathrm{I} .86}$ & $0.4^{8}$ & I. 37 \\
\hline \multirow{2}{*}{ I974. } & \multirow{2}{*}{$\cdot \cdot \cdot$} & \multirow{2}{*}{\multicolumn{3}{|c|}{$\cdot \cdot \cdot \cdot$}} & $\bar{d}$ & 2.29 & -23.29 & $-0.5 \mathrm{I}$ & 0.34 & 0.29 & 3.14 & 6.00 \\
\hline & & & & & $t$ & 0.72 & $4 \cdot 5^{8}$ & 0.26 & 0.63 & 0.12 & 2.01 & $2.7^{8}$ \\
\hline \multirow{2}{*}{ I 975 . } & \multirow{2}{*}{$\cdot \cdot \cdot$} & \multirow{2}{*}{$\cdot$} & \multirow{2}{*}{\multicolumn{2}{|c|}{$\cdot \cdot \cdot$}} & $\bar{d}$ & 0.00 & - 3.II & 0.98 & 0.06 & -0.22 & 0.00 & $2 . .00$ \\
\hline & & & & & $t$ & 0.00 & 0.35 & 0.39 & o. I 5 & O.I 5 & 0.00 & $\mathrm{I} .22$ \\
\hline \multirow{2}{*}{\multicolumn{2}{|c|}{ overall years }} & \multirow{2}{*}{\multicolumn{2}{|c|}{ • }} & \multirow{2}{*}{. } & $\bar{d}$ & -6.03 & I. 77 & -0.27 & -0.45 & 0.66 & 2.14 & 2.43 \\
\hline & & & & & $t$ & 1.76 & 0.42 & 0.24 & $1.96^{*}$ & 0.76 & $2.50^{*}$ & $2.47^{*}$ \\
\hline
\end{tabular}

(I) Four years only.

$\left.{ }^{*}\right)$ Significant at 5 per cent level (significance given only for totals within populations). 
TABLE 8

Analyses of variance within the two populations (production traits) with respect to the two genotypes

Analyse de variance dans les deux populations (caractères de production) pour les deux génotypes

a) Jouy population

\begin{tabular}{|c|c|c|c|c|c|c|}
\hline Sources of variation & $d f$ & S.M. & E.N. & E.W. & H.U. & S.Th. \\
\hline Between genotypes (G). . . & I & I 75.64 & $65 \cdot 5 \mathrm{I}$ & 0.00 & I. I 6 & 4.24 \\
\hline Between years $(\mathbf{Y}) \cdot \ldots \cdot \cdot \cdot \cdot$ & 2 & I $385.85^{* *}$ & $286.29^{* *}$ & $33^{8.47^{* *}}$ & $2.87^{*}$ & $71 \cdot 79^{* *}$ \\
\hline Interaction $\mathrm{G} \times y \cdot \ldots$ & 2 & 66.19 & 17.42 & 14.77 & $0.0 \mathbf{I}$ & 0.98 \\
\hline Residual . . . . . . . & 66 & I 79.46 & 77.64 & I 2.31 & I.08 & 8.76 \\
\hline
\end{tabular}

b) M99 population

\begin{tabular}{|c|c|c|c|c|c|c|}
\hline Sources of variation & $d f$ & S.M. (I) & E.N. & E.W. & H.U. & S.Th. \\
\hline Between genotypes (G). . . & $\mathbf{I}$ & $538.5 \mathrm{I}^{*}$ & I $85.0 \mathrm{I}$ & 0.30 & $4.96^{* *}$ & $5 \cdot 7^{8}$ \\
\hline Between years $(\mathbf{Y}) . . .$. . & 4 & $2016.16 * *$ & 45 Or. $57^{* *}$ & $37 \cdot 7 \mathrm{r}$ & $3 \cdot 43^{* *}$ & I6.73 \\
\hline$\overline{\text { Interaction } \mathrm{G} \times \mathrm{Y} . . .}$ & 4 & 265.46 & $972.39^{* *}$ & $7 \cdot 32$ & $\mathbf{1} .63$ & I0.47 \\
\hline Residual . . . . . . . . & 60 & I89. Io & 252.01 & I 8.09 & 0.69 & 10.47 \\
\hline
\end{tabular}

(I) For sexual maturity the degrees of freedom are as follows : r, 3, 3 and 52 for between genotypes, years, interaction and residual respectively.

(**) Significant at I p. Ioo level.

$\left({ }^{*}\right)$ Significant at 5 p. roo level.

\section{Discussion}

\section{I. - The " major gene " hypothesis for polydipsia}

a) Existence of two populations of individuals

To summarize the above mentioned results, in a first step a discrimination between two types of birds is made thanks to the marked asymmetry of withinyear distributions. There-after it appears that the distribution of each type, years and strains being pooled, is visibly unimodal (Fig. I).

The distribution of " normal " birds does not deviate significantly from normality $\left(\chi^{2}=3.78\right.$ for $6 \mathrm{~d}$. f., N. S.). Its mean value varies from ca 2.0 to 2.6 according to year and strain (season and strain being confounded), with an 
Correlations between the traits studied for the genot. Corrélations entre les caractères étudiés pour les génot.

\begin{tabular}{|c|c|c|c|c|c|c|c|c|}
\hline & WI & $\frac{W I}{F}$ & $\% \frac{W I}{B W}$ & $\overline{\mathrm{W}}$ & $\Delta \mathrm{W}$ & $\mathrm{E}$ & $\mathrm{F}$ & \\
\hline WI. & & $(+.768)^{* *}$ & $+.530^{* *}$ & $+.308^{* *}$ & +.124 & +.150 & $+.340^{* *}$ & \\
\hline$\frac{W I}{W} \cdot$ & $(+.795)^{* *}$ & & $+.588 * *$ & -.056 & +.0 or 6 & $-304^{*}$ & $-315^{* *}$ & \\
\hline$\% \frac{W I}{B W}$ & $\left(+\cdot 74^{8}\right)^{* *}$ & $+.692^{* *}$ & & $-35^{8 * *}$ & $-.287^{*}$ & $(+. \mathrm{I} \mathbf{I} 0)$ & -.150 & \\
\hline & +.076 & $-.240^{*}$ & -. $466^{* *}$ & & $+.43^{6 * *}$ & +.066 & $+.544^{* *}$ & $\begin{array}{l}\text { 二.15 } \\
\text { 二.13 }\end{array}$ \\
\hline $\begin{array}{l}\Delta \mathrm{W} . \\
\mathrm{E} .\end{array}$ & $\begin{array}{l}-.043 \\
-.046\end{array}$ & $\begin{array}{l}-.242^{*} \\
(-.408)^{* *}\end{array}$ & $\begin{array}{r}-.189 \\
-.170\end{array}$ & $\begin{array}{l}+.056 \\
+.210\end{array}$ & -.182 & -.252 & $\begin{array}{l}+.28 \mathrm{I}^{*} \\
+.46 \mathrm{I}^{* * *}\end{array}$ & $\begin{array}{l}-13 \\
(-.24\end{array}$ \\
\hline & -.099 & $(-.682)^{* *}$ & $-.39 \mathrm{I}^{* *}$ & $+.524^{* *}$ & $+.396^{* *}$ & $(+.604)^{* *}$ & & -.2 \\
\hline & -.083 & $-.437^{* *}$ & -.026 & +.047 & +.226 & +.008 & $+.5^{82} * *$ & \\
\hline & -.096 & $-.453^{* *}$ & -.093 & +.025 & $+.693^{* *}$ & -.094 & $+.62 \mathrm{I} * *$ & +.8 \\
\hline S.M. . & -.094 & $+.14^{\circ}$ & +.027 & 一.06I & -.022 & $(-.268)^{*}$ & -.230 & -. \\
\hline E.N.. & +.009 & $-.294^{*}$ & +.038 & +.064 & $-.2 \mathrm{II}$ & $+.734^{* *}$ & $+.457^{* *}$ & +.2 \\
\hline E.W. & -.144 & $-.242^{*}$ & -.224 & $(+.162)$ & .000 & 十.oII & +.160 & \\
\hline H.U. & +.048 & +.184 & -.059 & 一. .06I & -.170 & -.072 & -.146 & -.06 \\
\hline S.Th. & $(-.063)$ & $(-.050)$ & $(-. \mathbf{I} 3 \mathbf{I})$ & 一. .086 & -.219 & $+.247^{*}$ & $(-.039)$ & -.17 \\
\hline W.L. & $-.242^{*}$ & $-.33^{* * *}$ & -.193 & $+.245^{*}$ & 一.I9I & $(+.219)$ & $+.267^{*}$ & +.27 \\
\hline & +.052 & +.022 & $(-.009)$ & $+.52 \mathrm{I}^{* *}$ & -.028 & +.079 & +.117 & \\
\hline
\end{tabular}

Above the diagonal are the correlation figures for (Di) birds and those below are for (di). (69 d.f.)

$\left(^{*}\right)$ Significant at $5 \mathrm{p}$. Ioo level.

(**) Significant at $\mathrm{x}$ p. roo level.

Values significantly heterogenous for the 2 populations are in parentheses.

overall value of 2.28 . On the contrary, the distribution of " abnormals " is markedly asymmetric $\left(^{*}\right)$ and has a higher variance than the former $(.67$ vs .35 for " normal " hens). The overall mean of " abnormals" is 4.24 .

According to the means and standard deviations of the distribution of " normals " and " polydipsics", the probability for the distribution of one type containing individuals of the other type appears to be low enough so as not to bias appreciably the numbers of each of the two types.

\section{b) Test of the major gene hypothesis}

This likely existence of two distinct populations suggested in both populations the presence of several genotypes at a locus with " major" effect. The fact that families contained either only " normal " daughters, or a mixture of " normal" and " abnormal " ones with, on the whole, predominance of the former, suggests then the hypothesis of a single recessive being responsible for " polydipsia ", the

$\left(^{*}\right)$ Which may come for a small part from year means being based mainly on " normal " birds with a somewhat variable contribution from "abnormal" ones. 
di) and (Di) pooled on within population basis

di) et (Di) regroupés sur une base intra-population

\begin{tabular}{|c|c|c|c|c|c|c|c|}
\hline $\mathbf{R}^{\prime}$ & S.M. & E.N. & E.W. & H.U. & S.Th. & W.I. & S.I. \\
\hline $\begin{array}{l}+.110 \\
-.052\end{array}$ & $\begin{array}{r}-150 \\
+.079\end{array}$ & $\begin{array}{l}+.086 \\
-.236^{*}\end{array}$ & $\begin{array}{r}+.167 \\
+.083\end{array}$ & $\begin{array}{l}-.254^{*} \\
-.174\end{array}$ & $\begin{array}{r}+.163 \\
+.035\end{array}$ & $\begin{array}{r}+.219 \\
-.048\end{array}$ & $\begin{array}{l}+.24^{6 *} \\
+.13^{6}\end{array}$ \\
\hline -.IOI & +.008 & $(+.053)$ & -.084 & $(-.040)$ & $+.07 I$ & +.084 &.$- \mathrm{II} 7$ \\
\hline$-.22 \mathrm{r}$ & -. I94 & +.100 & +.157 & —. 147 & 十.100 & +.126 & $+.366 * *$ \\
\hline$+.46 \mathrm{I} * *$ & $(-.272)^{*}$ & -. .063 & $-.275^{*}$ & 一. I 49 & 一. I 43 & -.080 & $(-.023)$ \\
\hline$(-347)^{* *}$ & 一. .064 & $+.804^{* *}$ & $+.299^{*}$ & 一. $\mathrm{r}_{46} 6$ & $+.273^{*}$ & $\left(+\cdot 3^{28} 8\right)^{* *}$ & +.037 \\
\hline$(+.434)^{* *}$ & $-.269^{*}$ & $+.411 * *$ & $+.316^{* *}$ & $(-.244)^{*}$ & +.215 & $(+\cdot 394)^{* *}$ & +.179 \\
\hline$+.775^{* *}$ & -.055 & 一. I 83 & +.172 & -.074 & -.063 & +.013 & +.052 \\
\hline & 一.21 2 & 一.194 & $\begin{array}{l}+.070 \\
+\quad 88\end{array}$ & 一. I93 & $\begin{array}{l}\text { 一.080 } \\
+.325 * *\end{array}$ & $\begin{array}{l}-.023 \\
(-.250) *\end{array}$ & $\begin{array}{c}+.155 \\
+.77)\end{array}$ \\
\hline+ .oII & $\left(-.45^{6}\right)^{* *}$ & -.295 & -.033 & $\begin{array}{r}1.193 \\
-.193\end{array}$ & $\begin{array}{r}.025 \\
-.05 I\end{array}$ & $(+.278)^{*}$ & -.070 \\
\hline 十.III & $+.23^{8 *}$ & -.073 & & -. $15^{\circ}$ & $+.486^{* *}$ & +.202 & $+\cdot 388 * *$ \\
\hline-.142 & $+.250^{*}$ & -.160 & +.168 & & $-.25 \mathrm{I}^{*}$ & +.080 & -.120 \\
\hline$-.240^{*}$ & +.130 & -. II 7 & $+.269^{*}$ &.$+ \mathrm{II} 2$ & & +.177 & $+.45^{8 * *}$ \\
\hline+.084 & -.098 & +.230 & +.182 &.$+ \mathrm{IO}_{3}$ & $(-.149)$ & & +.155 \\
\hline-.092 & +.008 & -.005 & +.048 & +.012 & +.027 & +.102 & \\
\hline
\end{tabular}

majority of parents being either " normal " homozygotes or heterozygotes. Of course this hypothesis was suggested on the other hand by analogy with the proven existence of such a recessive gene in other populations (BUSS and MURPHY, I965).

In full-sib families where at least one " polydipsic " female was detected, the number of ,, normal " and " polydipsic " hens is respectively 72 vs 37 in the "Jouy" strain, 85 vs 43 in the "M99", and 157 vs 80 for both pooled, as has been already mentioned.

The expected numbers to which these figures have to be compared depend on the genotypes of the parents. Calling Di the dominant allele and di the postulated recessive allele causing high water intake (this being or not identical with that described by Buss and MURPHY), abnormal daughters can appear when the two parents are Didi or when one is Didi, the other didi (the case of two abnormal parents is expected to be rare and does not seem to have been realized).

The frequency of " abnormal " females on the whole is . I74 in the " Jouy" population and .II5 in the "M99 ". This corresponds to an estimated frequency for di $q=.42$ in the "Jouy" strain and $q=.34$ in the "M99". The expected frequency of heterozygotes is then respectively .48 and .44, which leads to estimate the proportion of the Didi and didi genotypes among parents having at least one di allele, respectively to .74 and .26 (for " Jouy ") and to 
TABI,

Correlations: test of heterogenei,

Corrélations : test d'hétérogénéit

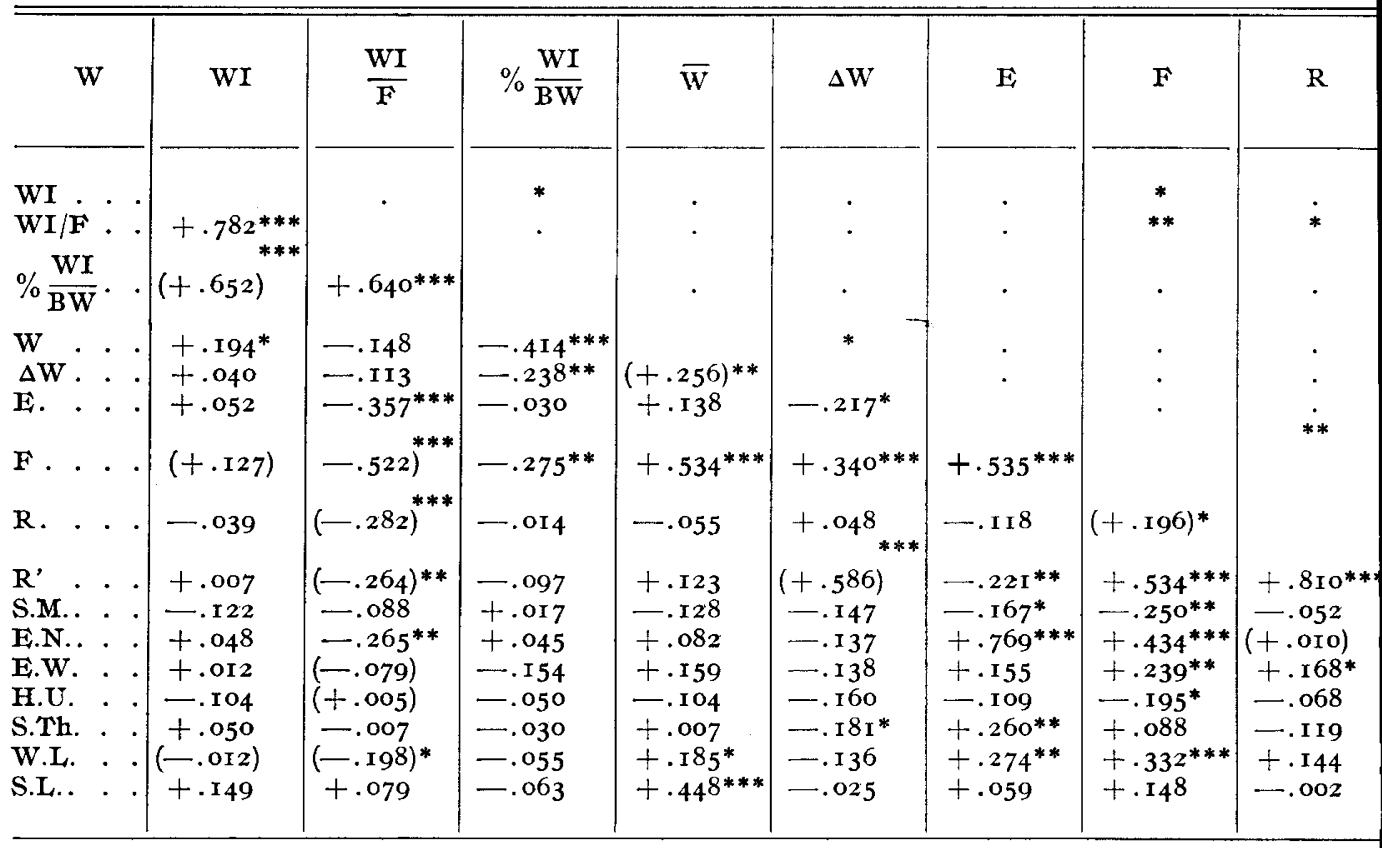

Under the diagonal are common estimates of the correlations (pooled within genotypes) and their significanc Values significantly different for the two genotypes are in parentheses.

Above the diagonal is the significance of heterogeneity between genotypes for the correlations $\left(^{*}=5 \mathrm{p}\right.$

.79 and .2I (for "M99"): from this the expected proportion of the matings susceptible of giving didi progeny (Didi $\times$ Didi or Didi $\times$ didi) may be estimated. Accordingly the expected ratio of progeny of (Di) and (di) phenotype on the whole would be 2.58 / I for " Jouy" and 2.48 / I for " M99", corresponding respectively to the expected numbers 78.6 and 30.4 in the first strain, 91.2 and 36.8 in the second. The difference with observed numbers is not significant, although some lack of (Di) appears (corrected $\chi^{2}=$ I.70 for " Jouy " and I.24 for " M99 ").

It must be added that the limited size of dam families (3.95 progeny on average) is one more factor tending to underestimate the proportion of (Di) progeny, as families with no didi progeny but corresponding to mating types capable of giving it are discarded in our procedure. This may be estimated to represent around 79 additional (Di) birds $(*)$, which would lead to the numbers 236 (Di) vs 80 (di) birds on total. Compared to the expected ratios estimated before

(*) Based on 4 daughters, the probability of no didi progeny in Didi $\times$ Didi matings is $3 / 4)^{4}$; in Didi $x$ didi matings it is $(I / 2)^{4}$. Each of these probabilities multiplied by expected frequency of mating type gives our gross estimation. 
veen genotypes and pooled estimates

e génotypes et estimations groupées

\begin{tabular}{|c|c|c|c|c|c|c|c|}
\hline $\mathrm{R}^{\prime}$ & S.M. & E.N. & E.W. & H.U. & S.Th. & W.L. & S.I. \\
\hline * & . & . & $(\dot{*})$ & * & . & * & $\dot{.}$ \\
\hline . & . & . & . & . & - & . & . \\
\hline$\therefore$ & . & . & . & . & . & . & - \\
\hline * & . & . & . & . & . & . & . \\
\hline . & . & . & . & . & - & . & - \\
\hline . & - & . & . & . & - & - & • \\
\hline . & - & $(*)$ & . & . & . & . & \\
\hline & - & . & . & . & . & . & - \\
\hline-.124 & & . & . & . & . & . & . \\
\hline-.092 & $-.378^{* * *}$ & & . & . & . & . & . \\
\hline $\begin{array}{l}.090 \\
-.168^{*}\end{array}$ & $+.213^{*}$ & -.053 & & . & $*$ & - & $*$ \\
\hline $\begin{array}{l}-.168^{*} \\
-.160\end{array}$ & $\begin{array}{l}+.153 \\
+.229^{* *}\end{array}$ & $\begin{array}{l}-.176^{*} \\
-.084\end{array}$ & $\begin{array}{l}+.009 \\
+.383^{* * *}\end{array}$ & $(-.070)$ & 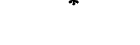 & . & ${ }^{*}$ \\
\hline+.054 & -. $174^{*}$ & $+.255^{* *}$ & $+.192^{*}$ & +.092 & +.014 & & * \\
\hline+.032 & +.063 & -.037 & $(+.224)^{*}$ & -.054 & $(+243)^{* *}$ & +.128 & . \\
\hline
\end{tabular}

$=\mathrm{r}_{3} 8$ d.f.).

level; $* *=$ I p. roo level.)

(giving $2.53 / \mathrm{I}$ for both strains together) this gives a non-significant chi-square of I.26. Finally the concordance between the hypothesis and available observations seems acceptable. The constitution of a line homozygote for the postulated di allele is being worked out so as to further confirm our hypothesis.

\section{2. - Means of genotypes and analyses of variance}

The wide difference between the two " genotypes " (di) and (Di) for the mean values within years given in table $3(a$ and $b)$ for the two populations and the overall means for water intake proves to be highly significant, this being confirmed by $t$-test and analysis of variance in tables 4 and 5 . The same holds true for percentage of water intake related to body weight.

The average values for water/feed ratio for (di), within populations and years, are about twice those of (Di). Meanwhile, the difference between " genotypes " for feed intake proved to be insignificant. That means that the variation between the two genotypes for water/feed ratio is essentially due to water intake. 
This finding is in agreement with the result pointed out by Buss and MurPHy (1965) on polydipsic birds. The variation, within our two populations, for water intake proves to be of the same order, and the same for water/feed ratio.

Both genotypes have no effect on body weight, gain in body weight and egg mass, their mean values being almost the same. On the other hand, the average difference between genotypes for the deviations of feed intake from regression on body weight, egg mass $\left(R^{\prime}\right)$ or from regression on these same two traits and in addition body weight variation $(R)$ prove also to be significant, with a lower mean value of (Di) birds as compared to (di), especially for $R$. It may be worthy to note, accordingly, a possibility of indirect selection on this last trait by discarding (di) birds, detected by their watery droppings.

There are no significant differences between the two genotypes for egg number and egg weight overall the estimates on the two populations. The mean values for wattle length prove to be significantly different, for both populations, but in opposite directions. Another difference, for shank length and Haugh units, is found only in the M99 population.

Finally, it is interesting to mention that no significant interaction is observed for any of the traits studied.

\section{3. - Correlation estimates}

As concerns the pooled estimates of correlations for the two populations, Table 9 shows that water/feed ratio is positively correlated, as expected, with both water intake and percentage of water to body weight for (Di) birds, and negatively correlated with feed intake, egg mass and egg number. The same can be observed for the estimates on the (di) birds; moreover, a negative correlation can be observed between water/feed ratio and body weight, variation in body weight, and also $R$ and $R^{\prime}$, egg weight and wattle length. Buss and MURPHY (I965) dis not find statistically significant correlations for water/feed ratio and egg number, egg weight, albumen quality, shell thickness or body weight.

Water intake is positively and highly significantly correlated with both body weight and feed intake for (Di) birds. On the other hand, shank length is correlated positively, also, with water intake, egg weight and shell thickness. Meanwhile haugh units correlate negatively with both water intake and shell thickness. The gain in weight and body weight are highly positively correlated for the (Di) birds but not for (di) genotype.

The correlations estimated on the (di) birds show that $\mathrm{R}$ and feed intake are highly positively correlated. $\mathrm{R}$ is positively correlated will wattle length while $R^{\prime}$ is negatively correlated with shell thickness. The same negative correlation is observed between water intake and wattle length. Haugh units for (di) genotype is positively correlated with sexual maturity.

The significant differences between the two genotypes, with respect to correlation estimates within each of them (table ro), are in some way genetic differences. This is the case for correlations between water intake and feed intake, between water/feed ratio and feed intake, $R, R^{\prime}$, egg weight, Haugh units and wattle length. These differences may be explained by the different variance in water intake between the two genotypes. Other correlations which appear significantly different between (Di) and (di) birds are those between body weight and variation in weight, between $\Delta \mathrm{W}$ and $\mathrm{R}^{\prime}, \mathrm{F}$ and $\mathrm{R}, \mathrm{EN}$ and $\mathrm{R}$, shell thickness and Haugh units, shell thickness and shank length, egg weight and shank length. It may be safe to confirm further these differences with more numerous data. 


\title{
Conclusion
}

From the above mentioned results, it appears that the excessive water intake found for some birds in our two populations is achieved by genetic factors including probably a major gene, with apparently similar effect to that described previously by Buss and Murphy (I965).

As mentioned above, the significant difference found in the present work between " normal " and " polydipsic" hens for R and $\mathrm{R}^{\prime}$ (characterizing the part of feed efficiency independent from body weight and egg production) may suggest selecting against the di allele in such populations as ours, for improvement of the $R$ trait, as our " polydipsic " birds are in general easily detected in cages according to their watery droppings.

This effect associated to the Di locus may partly or totally account for the overall positive correlation (ignoring any variation at a particular locus), found previously between water intake and $R$, at fixed total feed intake, in the "Jouy " population (BORDAS and MERAT, I974). On the other hand, the same remark as in this previous paper can be made as to a possible explanation of the effect of supplementary water intake on " residual " feed intake by the additional caloric requirement caused by the raise of temperature of ingested water to body temperature: estimating around $20^{\circ} \mathrm{C}$ the mean temperature of ingested water, this explanation can give account of only a limited part of the observed effect.

Rę̧u pour publication en juillet 1978 .

\section{Remerciements}

Nous remercions le $\mathrm{D}^{\mathrm{r}} \mathrm{A}$. Most AGEER, Cairo University, Le Caire, et le $\mathrm{D}^{\mathrm{r}} \mathrm{J}$. H. Van Middelkoop, Spelderholt Institute, Beekbergen, pourleurs utiles remarques à la lecture de ce manuscrit.

\section{Résumé}

\author{
Sur l'hérédité de l'ingestion d'eau \\ et de l'efficacité alimentaire chez la poule
}

Cette étude a porté sur deux populations, "M99 " et "Jouy ", pendant cinq années consécutives. Le but principal était de déterminer la variation génétique de l'ingestion d'eau et du rapport eau/aliment consommé et l'effet de ce rapport sur l'ingestion d'aliment. Les principaux résultats peuvent être résumés comme suit :

I. - Un gène majeur récessif dans les deux populations semble responsable d'une " polydipsie " chez certains individus. Il y a une différence moyenne importante d'ingestion d'eau entre les deux génotypes distingués ici, di di correspondant à une consommation d'eau excessive et les génotypes normaux étant désignés par (Di).

La valeur moyenne du rapport eau/aliment pour les poules di di se révèle être à peu près double de celle des poules (Di).

z. - La variabilité du rapport eau/aliment est essentiellement due à l'ingestion d'eau.

3. - Les oiseaux "polydipsiques" ont une consommation alimentaire " tésiduelle " (une fois tenu compte du poids corporel et de la production d'œufs) significativement plus élevée que celle des " normaux".

4. -- Il n'y a pas de différence significative entre les génotypes comparés pour le nombre et le poids des œufs.

5. - Certaines corrélations phénotypiques peuvent être modifiées par la présence du génotype di di. 


\section{References}

Bigrer B. W., EleAger T. H., BARNeitT B. D., I966. The effect of feed and water deprivation on water and feed consumption, body weight and mortality in broiler chickens of various ages. Poult. Sci., 45, I045.

BORdAS A., MERAT P., I974. Variabilité génétique et corrélations phénotypiques caractérisant la consommation alimentaire des poules pondeuses après correction pour le poids corporel et la ponte. Ann. Génét. Sél. anim., 6, 369-379.

BrEth, F. E., I964. L'eau et les volailles. La Revue de l'Élevage, 11, I2 I5.

Buss E. G. MURPHY R. R., I965. Method of determination, assessment of possibly associated traits and evidence for genetic influence of excessive water intake by chickens. Poult. Sci., 44, $135^{8 .}$

LEeson S., SUmmers J. D. and Moran E. T., Jr, 1976. Avian water metabolism. A review. World's Poult. Sci. J., 32, 185-195.

ZEIGLER H. P., GREEN H. L., SIEGEL J., 1972. Food and water intake and weight regulation in pigeon. Physiol. Behav., 8, 127. 